\title{
The low-frequency radio emission and spectrum of the extended SNR W44: new VLA observations at 74 and $324 \mathrm{MHz}$
}

\author{
G. Castelletti ${ }^{1, \star}$, G. Dubner ${ }^{1, \star \star}$, C. Brogan ${ }^{2}$, and N. E. Kassim ${ }^{3}$ \\ 1 Instituto de Astronomía y Física del Espacio (IAFE), CC 67, Suc. 28, 1428 Buenos Aires, Argentina \\ e-mail: gcastell@iafe.uba.ar \\ 2 National Radio Astronomy Observatory, 520 Edgemont Road, Charlottesville, VA 22903, USA \\ 3 Remote Sensing Division, Code 7213, Naval Research Laboratory, 4555 Overlook Avenue, SW, Washington DC, USA
}

Received 5 January 2007 / Accepted 1 March 2007

\begin{abstract}
Aims. We present new Very Large Array (VLA) radio images at 74 and $324 \mathrm{MHz}$ of the SNR W44. The VLA images, obtained with unprecedented angular resolution and sensitivity for such low frequencies (HPBW 37" at 74 MHz, and 13" at 324 MHz), have been used in combination with existing $1442 \mathrm{MHz}$ radio data, Spitzer IR data, and ROSAT and Chandra X-ray data to investigate morphological and spectral continuum properties of this SNR.

Methods. The observations were carried out with the VLA simultaneously at 74 and $324 \mathrm{MHz}$ in the A and B configurations and at $324 \mathrm{MHz}$ in the $\mathrm{C}$ and $\mathrm{D}$ configurations. The radio continuum spectral index distribution was derived through direct comparison of the combined data at 74, 324, and $1442 \mathrm{MHz}$. In addition, to isolate and identify different spectral components, tomographic spectral analysis was performed.

Results. We measured total flux densities of $634 \mathrm{Jy}$ and $411 \mathrm{Jy}$ at 74 and $324 \mathrm{MHz}$, respectively, for W44, and from a careful assessment of published values between 22 and $10700 \mathrm{MHz}$ derived a global integrated continuum spectral index $\alpha=-0.37 \pm 0.02$. The spatially resolved spectral index study revealed that the bright filaments, both around and across the SNR, have a straight spectrum between 74 and $1442 \mathrm{MHz}$, with $\alpha \sim-0.5$, with two clear exceptions: a short portion of the SNR limb to the southeast, with $\alpha$ varying between 0 and +0.4 and a bright arc to the west where the spectrum breaks around $300 \mathrm{MHz}$ and becomes concave down. We conclude that at the shell and along the internal filaments, the electrons responsible for the synchrotron emission were accelerated at the shock according to a simple diffusive shock model. The positive spectrum corresponds to a location where the SN shock is running into a molecular cloud and the line of sight intersects the photo dissociation region of an HII region and a young stellar object is present. Such spectral inversion is a classic signature of thermal absorption, either from ionized gas in the postshock region, from the HII region itself, or both. The curved spectrum on the westernmost bright arc is explained as the consequence of strong post-shock densities and enhanced magnetic fields after the interaction of the SN shock with a coincident molecular cloud. No spectral index trace was found indicating any connection between the associated pulsar PSR B1953+0.1 and the surrounding shell, nor between the SNR and the 3EG $1853+0114 \gamma$-ray source proposed to be associated with W44. The comparison of the $324 \mathrm{MHz}$ image with a $4.5 \mu \mathrm{m}$ IR image obtained with Spitzer underscored an impressive correspondence between emission both to the north and west sides of W44, while the comparison with ROSAT and Chandra images confirm that the synchrotron radio emission surrounds the thermal X-ray radiation.
\end{abstract}

Key words. ISM: individual objects: W44 - ISM: supernova remnants - radio continuum: ISM

\section{Introduction}

The interaction of supernova remnant (SNR) shocks with inhomogeneous interstellar gas strongly affects the spatial and temporal evolution of SNRs. The density distribution of the ambient circumstellar material (CSM) and interstellar medium (ISM) can influence the formation of a variety of SNR morphologies, including specific sub-classes of SNRs such as "thermal-X-ray composites" (also called mixed-morphology SNRs, Rho \& Petre 1998), or remnants with bilateral symmetry (Gaensler 1998). In this respect, sensitive high-resolution radio observations of SNRs are particularly useful for studying the physics of shock waves traveling through nonuniform environments in the ISM.

On the other hand, continuum radio spectra made accurate by extension to long wavelengths provide sensitive and often unique means of distinguishing between different physical

\footnotetext{
^ Post-Doc Fellow of CONICET, Argentina.

$\star \star$ Member of the Carrera del Investigador Científico of CONICET, Argentina.
}

processes taking place either within SNRs (e.g. shock acceleration), in their immediate surrounding, or in the ISM along the line of sight (e.g. thermal absorption, Lacey et al. 2001; Brogan et al. 2005). Based on accurate spectral continuum index studies it is possible to constrain shock acceleration theories that predict subtle variations in both spatially resolved (Anderson \& Rudnick 1993) and integrated spectra (Reynolds \& Ellison 1992). In addition, the information provided by low frequency observations of SNRs is a powerful complement to higher frequency radio, $\mathrm{X}$-ray, and infrared (IR) data to study the complex processes associated with SNR shocks (Brogan et al. 2005).

W44 (G34.7-0.4) is a bright radio SNR $\left(S_{1 \mathrm{GHz}} \sim 230 \mathrm{Jy}\right)$ with an asymmetric morphology, about 0.5 in size, and located in a complex region of the inner Galactic plane rich in both thermal and nonthermal sources. W44 is an archetype of the "mixedmorphology" class of remnants, characterized by a highly filamentary radio shell and centrally concentrated thermal X-ray emission. Morphological studies of this remnant in the optical band reveal Balmer-dominated, [NII], and [SII] filaments from 
radiative shocks, with some concordance with the radio emission (Rho et al. 1994; Giacani et al. 1997).

This SNR is also of interest because it constitutes one of the few demonstrated cases of a SNR-molecular cloud interacting system. Seta et al. (1998) observed six giant molecular clouds that appear to be partially surrounding the remnant. Higher spatial resolution ${ }^{12} \mathrm{CO}$ observations of the full extent of W44 presented by Seta et al. (2004) revealed that some of these clouds (at $v_{\text {LSR }} \sim 48 \mathrm{~km} \mathrm{~s}^{-1}$ ) are physically interacting with the remnant on its southeastern and western sides. These observations also show that the shocked molecular gas is confined to compact $(\sim 1.5 \mathrm{pc})$ cores located adjacent to bright radio filaments or knots. The physical interaction of the blast wave with the clumpy ISM is also supported by the detection of bright $\mathrm{OH}(1720 \mathrm{MHz})$ masers (at LSR velocities between $\sim 43$ and $\sim 47 \mathrm{~km} \mathrm{~s}^{-1}$, Hoffman et al. 2005, and references therein), and IR cooling lines from $\mathrm{H}_{2}$ (Reach et al. 2006).

The associated pulsar PSR B1853+01 is located inside the W44 shell, about $9^{\prime}$ south of the geometric center of the remnant. A characteristic age of about $2 \times 10^{4}$ years has been estimated for this pulsar (Wolszczan et al. 1991). Frail et al. (1996) and Petre et al. (2002) report the detection of a radio and X-ray nebula powered by an associated pulsar wind.

It has also been proposed that the EGRET source 3EG 1856+0114 is associated with W44 (Esposito et al. 1996). The $95 \%$ confidence contour for this $\gamma$-ray source coincides with the southeastern sector of the remnant (Thompson et al. 1996). de Jager \& Mastichiadis (1997) explained the observed GeV $\gamma$ rays in terms of relativistic bremsstrahlung and inverse Compton scattering produced by a power-law distribution of relativistic electrons injected by the pulsar PSR B1853+01. While this constitutes a possible explanation, a significant part of the $\gamma$-ray flux observed may also be due to SNR shock-cloud interactions (Fatuzzo \& Melia 2005). No $\gamma$-ray radiation at TeV energies was detected towards W44 using either the Whipple (Leslard 1995) or CANGAROO (Buckley et al. 1998) telescopes, suggesting a spectral cutoff between $\mathrm{GeV}$ and $\mathrm{TeV}$ energies (Rowell et al. 2000).

A distance of about $3 \mathrm{kpc}$ was first suggested for W44 by Radhakrishnan et al. (1972) and Caswell et al. (1975) based on $\mathrm{H}$ I absorption measurements. From the afore mentioned molecular studies revealing associated gas with LSR velocities between approximately 43 and $48 \mathrm{~km} \mathrm{~s}^{-1}$ and using a flat rotation curve with the solar constants of $R_{0}=7.6 \mathrm{kpc}$ (Eisenhauer et al. 2005) and $\Omega_{0}=27.2 \mathrm{~km} \mathrm{~s}^{-1} \mathrm{kpc}^{-1}$ (Feast \& Whitelock 1997), a kinematical distance of $2.9 \pm 0.2 \mathrm{kpc}$ can be derived for W44. Thus W44 is probably an object of the Sagittarius arm consistent with it being a core-collapse $\mathrm{SNe}$ from a massive progenitor star.

In this paper we present the highest resolution and sensitivity images of the SNR W44 yet obtained at low radio frequencies. These images were generated from multiple-configuration Very Large Array (VLA) ${ }^{1}$ observations at 74 and $324 \mathrm{MHz}$ carried out in 2002 and 2003. Based on the comparison of the new images with existing VLA data at $1442 \mathrm{MHz}$, we have performed the first detailed study of the radio continuum spectral properties of W44, searching for changes in spectral index as a function of frequency and position. In addition, special attention was paid to the comparison between our high resolution radio images and the best existing infrared data from Spitzer and in X-rays from ROSAT and Chandra.

1 The Very Large Array of the National Radio Astronomy Observatory is a facility of the National Science Foundation operated under cooperative agreement by Associated Universities, Inc.
Table 1. Observational summary.

\begin{tabular}{lccc}
\hline \hline $\begin{array}{l}\text { Observing } \\
\text { dates }\end{array}$ & $\begin{array}{c}\text { VLA } \\
\text { Config. }\end{array}$ & $\begin{array}{c}\text { Bandwidth } \\
(\mathrm{MHz})\end{array}$ & $\begin{array}{c}\text { Integration } \\
\text { time (h) }\end{array}$ \\
\hline \multicolumn{4}{c}{ 74 MHz Parameters } \\
\hline 2003 Aug. 31 & $\mathrm{A}$ & 1.562 & 7.5 \\
2003 Sep. 1 & $\mathrm{A}$ & 1.562 & 2.6 \\
2002 Jun. 15 & $\mathrm{B}$ & 1.562 & 6.0 \\
\hline \multicolumn{4}{c}{ 324 MHz Parameters } \\
\hline 2003 Aug. 31 & $\mathrm{A}$ & 3.125 & 7.5 \\
2003 Sep. 1 & $\mathrm{A}$ & 3.125 & 2.6 \\
2002 Jun. 15 & $\mathrm{B}$ & 3.125 & 6.0 \\
2002 Dec. 14 & $\mathrm{C}$ & 3.125 & 4.0 \\
2003 Mar. 16 & $\mathrm{D}$ & 3.125 & 6.0 \\
\hline
\end{tabular}

\section{Observations and data reduction}

We have performed low frequency observations of the SNR W44 at 74 and $324 \mathrm{MHz}$ using multiple-configurations of the VLA. Table 1 summarizes various observing details. Data at both frequencies were obtained in spectral-line mode to enable radiofrequency interference (RFI) excision and to mitigate bandwidth smearing. The observations at $74 \mathrm{MHz}$ and $324 \mathrm{MHz}$ were performed with 64 and 32 channels, respectively. A model image of Cygnus A was used to correct the bandpass ${ }^{2}$. The absolute amplitude scale was set using Cygnus $\mathrm{A}$ at $74 \mathrm{MHz}$ and $3 \mathrm{C} 48$ at $324 \mathrm{MHz}$. Also, for the $324 \mathrm{MHz}$ data, regular observations of the secondary calibrator 1822-096 were used for initial phase and amplitude calibration. At both frequencies, the data from each array were fully reduced and imaged separately using the NRAO Astronomical Image Processing Software (AIPS) package. After initial calibration the data at 74 and $324 \mathrm{MHz}$ were averaged down to fourteen and eight channel spectral resolution data cubes, respectively, before imaging.

Two additional difficulties associated with low frequency radio observations are the wide-field imaging requirement imposed by the large field of view (the primary beam of the 324 and $74 \mathrm{MHz}$ data are 2.5 and 11.5 , respectively), and ionosphericbased phase variations that decorrelate phases on longer baselines, especially at $74 \mathrm{MHz}$ (Kassim et al. 1993). We therefore employed wide-field imaging as implemented in the AIPS task IMAGR, based on a pseudo-three-dimensional multifacet algorithm to deal with the non-coplanarity of the visibilities (Cornwell \& Perley 1992). Secondly, to remove ionosphericinduced phase distortions we employed several loops of selfcalibration to the data from each configuration separately at 74 and $324 \mathrm{MHz}$ (Cornwell \& Fomalont 1999). We note that angleinvariant self-calibration (as currently implimented in AIPS) is not sufficient to correctly compensating for ionospheric effects across the full field of view, especially at $74 \mathrm{MHz}$ (Cotton et al. 2004). However, self-calibration is adequate in the special case where the target source is located at the phase center and is the brightest source in the field, as is this case with W44.

The final calibrated $324 \mathrm{MHz}$ visibility data from the A, $\mathrm{B}, \mathrm{C}$, and D configurations were combined into a single $u v$ data set and imaged using a multi-scale CLEAN algorithm with four different scale sizes. For the final image, all facets were reassembled into one large field with the task FLATN within AIPS, resulting in a single image with an angular resolution of $13^{\prime \prime} 3 \times 13^{\prime \prime}, 2, \mathrm{PA}=-4.7$, and an rms noise of $5 \mathrm{mJy} \mathrm{beam}^{-1}$.

\footnotetext{
2 See http://lwa.nrl.navy.mil/tutorial/ to obtain source models in FITS format.
} 


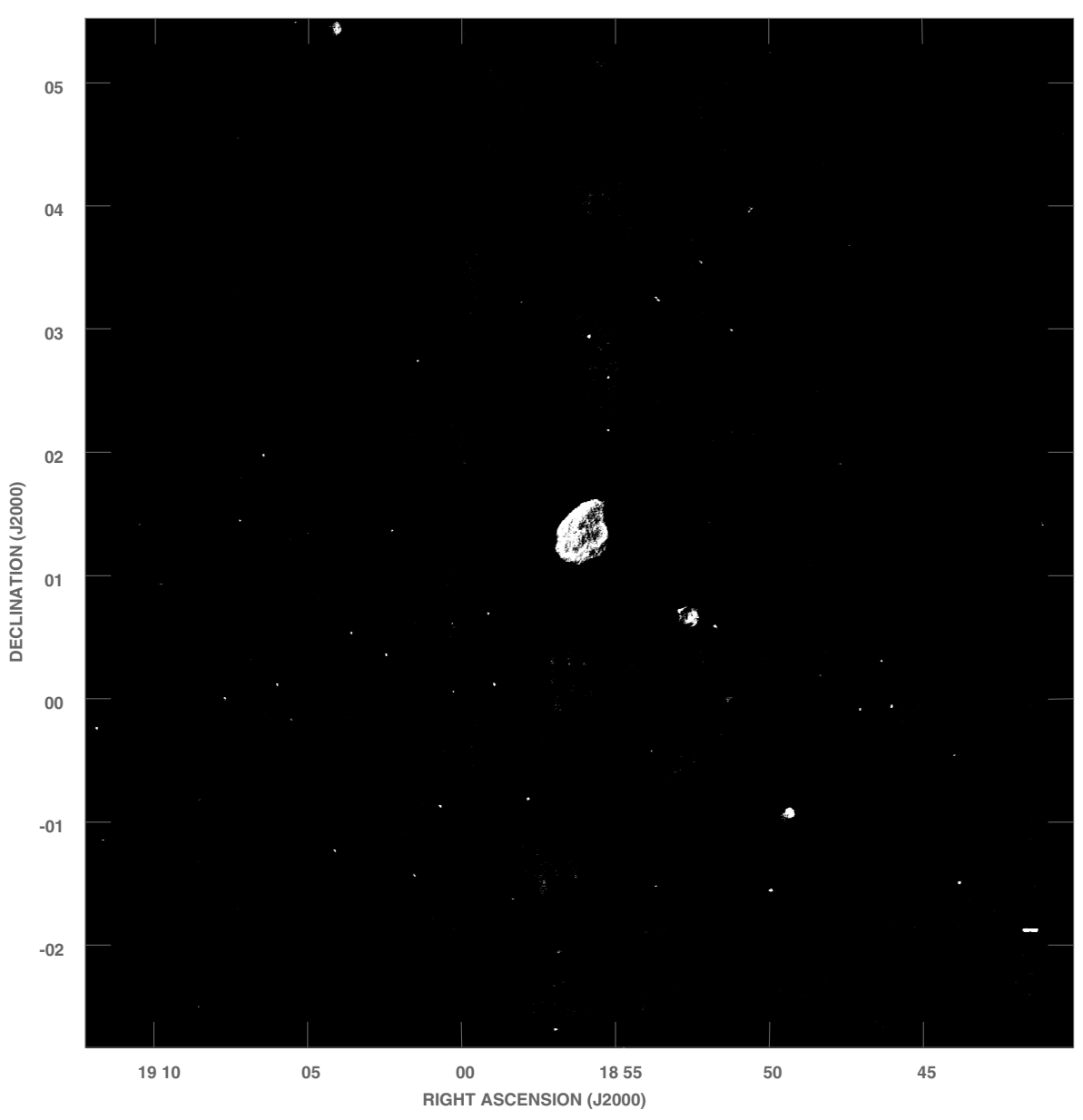

Fig. 1. This image shows a portion of the primary beam of the VLA at $74 \mathrm{MHz}$ centered on the SNR W44, after the combination of Aand B-configuration data. The image displayed does not include primary beam correction. The field covers $\sim 7.5$ square degrees at an angular resolution of $39^{\prime \prime} \times 36^{\prime \prime}\left(\mathrm{PA}=-47^{\circ}\right)$. The $\mathrm{rms}$ noise is $65 \mathrm{mJy}^{\text {beam }}{ }^{-1}$.
Data reduction and imaging at $74 \mathrm{MHz}$ were performed following the procedures outlined above ${ }^{3}$. For the A and B configuration $74 \mathrm{MHz}$ data, an initial phase-only self-calibration was conducted using the B-configuration $324 \mathrm{MHz}$ image as the starting model. The A and B configuration $74 \mathrm{MHz}$ data sets were then phase-only self-calibrated; the B configuration data also required a final amplitude self-calibration. The individually calibrated A and B data sets were then concatenated, and followed by several more rounds of self-calibration. The final image has a synthesized beam of $399^{\prime} 1 \times 35^{\prime \prime} \cdot 9$, PA $=-47^{\circ}$ and an rms noise of $65 \mathrm{mJy}^{\text {beam }}{ }^{-1}$.

A concern with self-calibrated images is that they can lose absolute position information subject to the arbitrary position of the starting model, which in this case may also be subject to ionospheric refraction. In order to check the astrometry in our 74 and $324 \mathrm{MHz}$ images, we compared the position of several small-diameter sources detected in these images with their positions from the NRAO VLA Sky Survey (NVSS). This catalog has an astrometric accuracy better than $1^{\prime \prime}$ in both RA and Dec for bright sources (Condon et al. 1998). We found and corrected for an average ionospheric induced refraction of $0.63^{\mathrm{s}} \pm 0.12^{\mathrm{s}}$ in RA and 9.' $1 \pm 1 . .6$ in Dec at $74 \mathrm{MHz}$ and $0.14^{\mathrm{s}} \pm 0.05^{\mathrm{s}}$ in RA and 3.'52 \pm 0. '93 in Dec at $324 \mathrm{MHz}$.

A high resolution VLA image of W44 at $1442 \mathrm{MHz}$ was produced from interferometric data taken in 1984 and 1985 (Jones et al. 1993) plus short spacing information from the Bonn $100 \mathrm{~m}$ $1408 \mathrm{MHz}$ Survey (Reich et al. 1990). This $1442 \mathrm{MHz}$ image

3 See at http://lwa.nrl.navy.mil/tutorial/ for a full description on $74 \mathrm{MHz}$ VLA data reduction. recovers a total flux density of $S_{1.4 \mathrm{GHz}}=300 \pm 7 \mathrm{Jy}$ and has a resolution and rms noise of $23^{\prime \prime} \times 19^{\prime \prime}$ and $4 \mathrm{mJy} \mathrm{beam}^{-1}$, respectively.

To study the spectral properties of W44 (Sect. 4), special care was taken to match the $u v$ coverage for the 74, 324, and $1442 \mathrm{MHz}$ VLA data. This was achieved by applying specific weighting schemes to the $324 \mathrm{MHz}$ data in order to provide the same resolution as the 74 and $1442 \mathrm{MHz}$ images, respectively. Similarly, we re-imaged the $1442 \mathrm{MHz}$ data so that they matched the $74 \mathrm{MHz}$ observations. Primary beam corrections were applied to the new 74 and $324 \mathrm{MHz}$ images before estimating flux densities.

\section{Results}

\subsection{VLA low frequency images of W44}

Figure 1 shows our $\sim 37^{\prime \prime}$ resolution VLA $74 \mathrm{MHz}$ image of a portion ( $\sim 7.5$ square degrees) of the large field of view centered on the SNR W44. Note that while sources are detected across the full field-of-view, only those located near the field center are correctly imaged by the angle-invariant self-calibration technique implimented here. A close-up view of the radio emission at $74 \mathrm{MHz}$ from W44 is shown in Fig. 2. This is the first subarcminute resolution image of this source ever obtained below $100 \mathrm{MHz}$. Though it is not sensitive to structures larger than $\sim 37^{\prime}$, it is sufficient to recover all information for this source about $30^{\prime}$ in size. Moreover, the fact that no negative bowl is apparent around the SNR at this frequency confirms that most of the large-scale structure is recovered. However, since W44 is 
almost at the limit of the largest imaged structures, some flux might still be missing from the $74 \mathrm{MHz}$ image.

In Fig. 3 we present the new VLA image of W44 at $324 \mathrm{MHz}$ with a resolution of $13^{\prime \prime}$ over a field of view of $\sim 2.5$ square degrees. A close-up view showing the detailed radio morphology of W44 at $324 \mathrm{MHz}$ is shown in Fig. 4. The present image has a beam size 300 times better and is 80 times more sensitive than any previously published $330 \mathrm{MHz}$ image of W44 (Kassim 1992). The combination of all four VLA array configurations is sensitive to structures between $13^{\prime \prime}$ and $1^{\circ}$ in size, ensuring complete sampling of all the spatial scales of the W44 emission. The high dynamic range achieved in these new observations has served to reveal new details previously unnoticed in W44, especially extreme filamentary and clumpy emission that is immersed in more tenuous diffuse emission.

At low radio frequencies the remnant has the appearance of a noncircular shell elongated in the southeast-northwest direction, with an average diameter of $\sim 30^{\prime}$ (or $25 \mathrm{pc}$ at the distance of $2.9 \mathrm{kpc}$ adopted in this paper). The brightest emission occurs along the eastern boundary, a region where previous observations have indicated the presence of molecular clouds probably interacting with W44. In the $324 \mathrm{MHz}$ image (Fig. 4) it is apparent that the east limb is not sharp. On the contrary, faint emission is clearly detected at a $5 \sigma$ level extending about $1^{\prime}$ beyond the bright rim. A similar weak radio halo was observed along the eastern side of the SNR Puppis A (Castelletti et al. 2006), a direction where Puppis A is encountering a molecular cloud as is the case for W44. To the west, the most outstanding feature is the short bright arc located near $18^{\mathrm{h}} 55^{\mathrm{m}} 20^{\mathrm{s}}, 01^{\circ} 22^{\prime}$ (J2000). The rest of the boundary is more diffuse in appearance.

Based on the new radio observations we have estimated integrated flux densities of $634 \pm 70 \mathrm{Jy}$ at $74 \mathrm{MHz}$ and $411 \pm 50 \mathrm{Jy}$ at $324 \mathrm{MHz}$ for $\mathrm{W} 44$, where the attenuation of the primary beam has been corrected for these estimates. The quoted errors take into account the statistical errors as well as uncertainties in the true extension of the SNR.

In the new sensitive $324 \mathrm{MHz}$ image, the low frequency counterpart of the pulsar wind nebula (PWN) around PSR B1853+01 discovered at higher radio frequencies and in X-rays (Frail et al. 1996; Petre et al. 2002), is clearly detected (small white cometary feature extending to the north of the pulsar position indicated by the plus sign in Fig. 4). An enlargement of the emission at $324 \mathrm{MHz}$ associated with this PWN is shown in Fig. 5. Similar to the $1.4 \mathrm{GHz}$ image shown by Frail et al. (1996), at $324 \mathrm{MHz}$ the pulsar is located at the apex of an elongated structure $\sim 2$ '.5 in extent. The two maxima observed inside the PWN, where the intensity peaks up to 56 mJy beam ${ }^{-1}$, were previously reported by Jones et al. (1993) as discrete sources. The integrated flux density of the PWN at $324 \mathrm{MHz}$ is $0.35 \pm 0.12 \mathrm{Jy}$. Because of the relatively lower sensitivity and angular resolution, combined with the typically flat radio spectra of all PWN, the W44 PWN is not apparent in the $74 \mathrm{MHz}$ image.

\section{The radio spectral index behavior}

\subsection{Integrated radio spectrum}

We have used the new estimated total flux densities at 74 and $324 \mathrm{MHz}$ together with data from the literature to determine the mean spectral index of W44. To construct accurate spectra of individual SNRs it is necessary that all flux densities be tied to the same absolute flux density scale. In Table 2 we list all integrated flux density estimates for W44 between 22 and $10700 \mathrm{MHz}$.

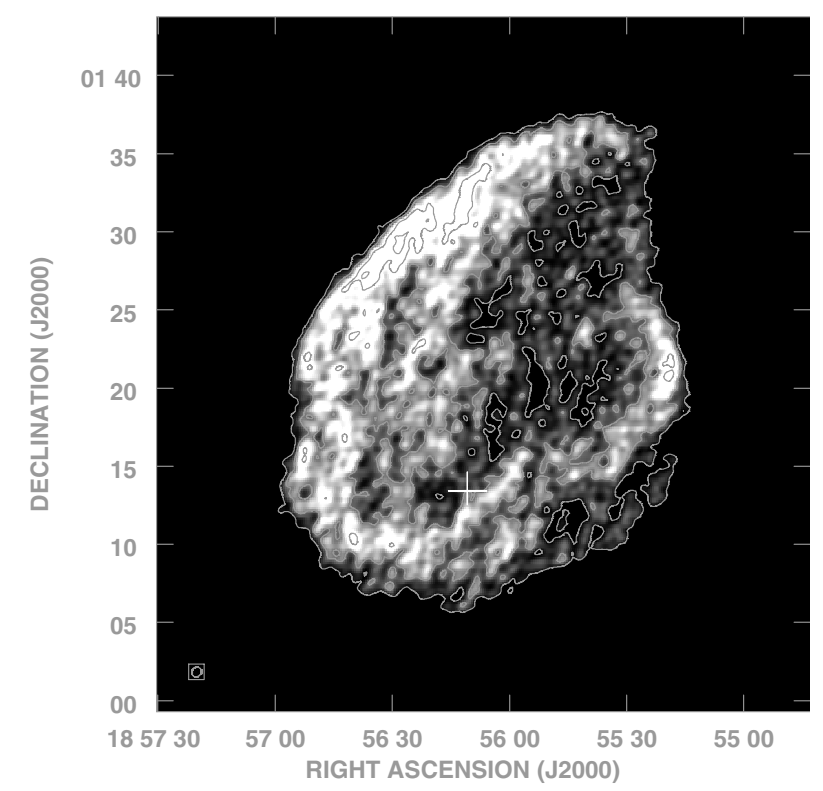

Fig. 2. Radio continuum emission at $74 \mathrm{MHz}$ from the SNR W44. The grayscale flux is linear ranging from 260 to $700 \mathrm{mJy} \mathrm{beam}^{-1}$. The rms noise level is $65 \mathrm{mJy}$ beam $^{-1}$, and the contours are traced at multiples of the $4 \sigma$ noise level, $0.260 \times(1,1.76,3.30,4.46) \mathrm{Jy} \mathrm{beam}^{-1}$. The synthesized beam shown at the bottom left corner is $39^{\prime \prime} \times 36^{\prime \prime}$. The white plus sign to the south of W44 indicates the position of the PSR B1853+01.

Most of the measurements over the frequency range between 408 and $10700 \mathrm{MHz}$ were extrapolated to the Baars et al. (1977) absolute flux scale for consistency. In some cases an estimate of the correction factor was not available because the original reference did not list the assumed flux densities of the primary calibrators. Below $408 \mathrm{MHz}(\log v \sim 2.6)$, where the systematic error of the Baars et al. scale is more than $\sim 5 \%$, no scaling was applied. These last values have been nevertheless used for the spectral fitting since they do not show a large spread.

In Fig. 6 we show a plot of the spectrum for W44 in which our new flux density determinations at 74 and $324 \mathrm{MHz}$ are indicated by filled circles. A single power law slope $\alpha=-0.37 \pm 0.02$ $\left(S_{v} \propto v^{\alpha}\right)$ adequately fits the flux densities measured over four decades in frequency without any obvious break. This integrated spectral index is quite flat for a typical shell type SNR, and agrees well with earlier estimates presented by Kassim (1989a,b, 1992) who derived a spectral index between $-0.4<\alpha<-0.3$. This result confirms Kassim (1989a)'s contention that the integrated spectrum of W44 remains a power law to well below $100 \mathrm{MHz}$. This behavior is in contrast to a number of other SNRs that exhibit a spectral turnover due to free-free absorption (e.g. W49B and 3C391, Lacey et al. 2001; Brogan et al. 2005). The fact that the new $74 \mathrm{MHz}$ integrated flux falls nicely on the power law confirms that our $74 \mathrm{MHz}$ image is missing little, if any emission on the largest spatial scales.

\subsection{Local variations in the radio spectral index}

We aim to discern whether the spectrum is a global property of this SNR, or if it is locally affected by the shock-ISM interaction and/or the presence of the pulsar in its interior. To investigate this, we performed a detailed study of the variations in the spectrum as a function of frequency and position within the remnant. For this analysis we have used the new high resolution images 


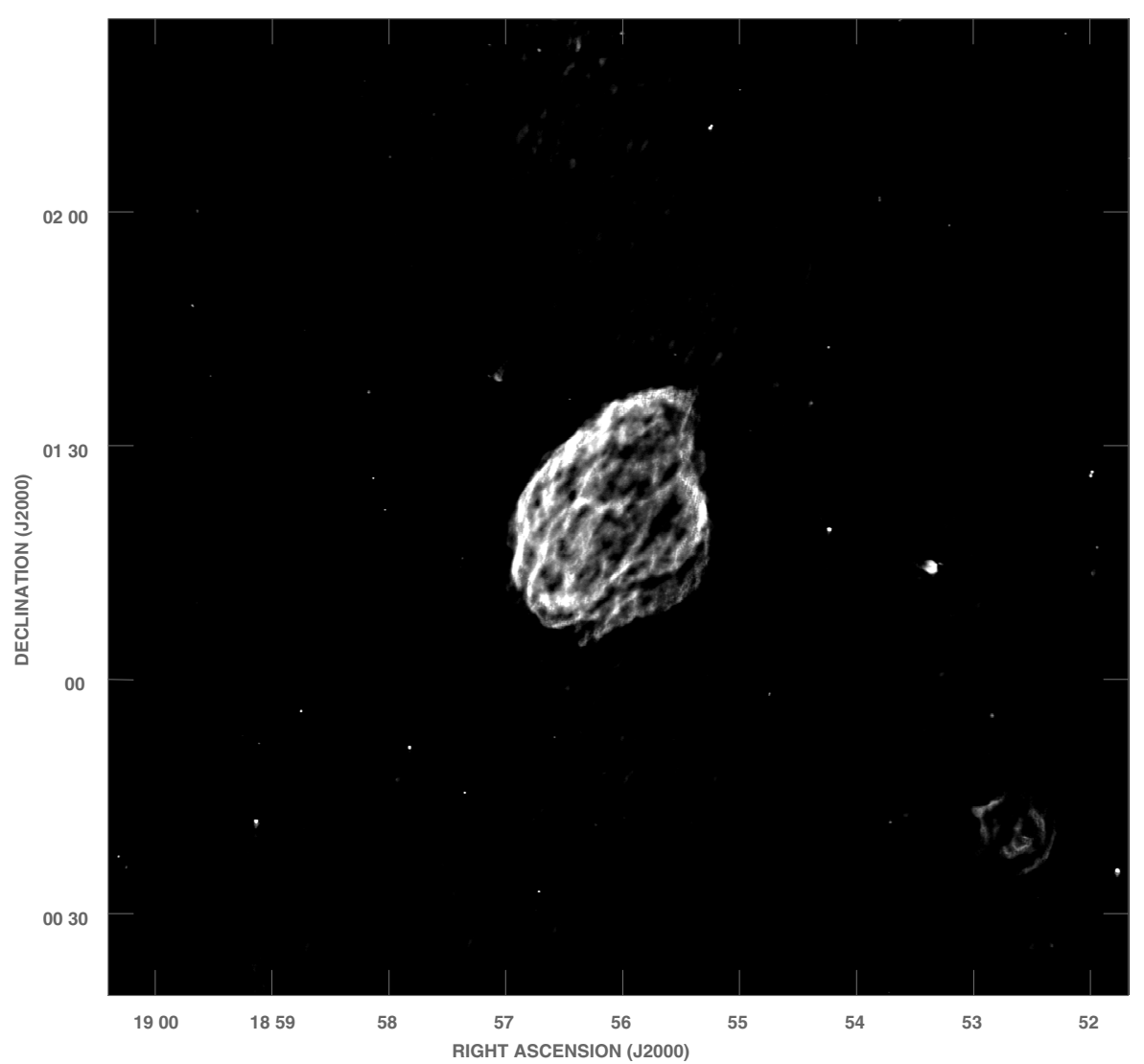

Fig. 3. An image of the field of view around the SNR W44 at $324 \mathrm{MHz}$. The image displayed does not include primary beam correction. The grayscale varies linearly between 15 and $70 \mathrm{mJy}^{\mathrm{beam}}{ }^{-1}$. The angular resolution is about $13^{\prime \prime} \times 13^{\prime \prime}$ with a position angle of -4.7 and the sensitivity level is $5.0 \mathrm{mJy}$ beam $^{-1}$. The source located near the bottom right corner $(\sim 185230,+0020)$ is the SNR Kes 79.

Table 2. Integrated flux densities on the SNR W44.

\begin{tabular}{|c|c|c|c|c|c|}
\hline $\begin{array}{l}\text { Frequency } \\
(\mathrm{MHz})\end{array}$ & $\begin{array}{l}\text { Scaled flux } \\
\text { density (Jy) }\end{array}$ & References & $\begin{array}{l}\text { Frequency } \\
(\mathrm{MHz})\end{array}$ & $\begin{array}{l}\text { Scaled flux } \\
\text { density (Jy) }\end{array}$ & References \\
\hline $22 \ldots$ & $881 \pm 108^{a}$ & Roger et al. (1986) & $750 \ldots \ldots$ & $270 \pm 27$ & Pauliny-Toth et al. (1966) \\
\hline 30.9 & $849 \pm 170^{a}$ & Kassim (1989b) & 960. & $208 \pm 8$ & Harris (1962) \\
\hline $38 \ldots$ & $766 \pm 230^{a}$ & Kassim (1989b) & 960 . & $230 \pm 16^{c}$ & Kuz'min (1962) \\
\hline $74 \ldots$ & $634 \pm 70^{b}$ & This work & 960. & $266 \pm 40$ & Wilson (1963) \\
\hline $83 \ldots$ & $500 \pm 27^{a}$ & Kovalenko et al. (1994) & 1390. & $173 \pm 35$ & Westerhout (1958) \\
\hline $85 \ldots$ & $436 \pm 131^{a}$ & Mills et al. (1958) & $1400 .$. & $188 \pm 23$ & Pauliny-Toth et al. (1966) \\
\hline 102. & $434 \pm 70^{a}$ & Kov & 400 . & $173 \pm 26$ & Kellermann et al. \\
\hline 111. & $500 \pm 70^{a}$ & enko & 1410. & $236 \pm 47$ & $r(1963)$ \\
\hline 159. & $680 \pm 122^{a}$ & Edge et a & 410. & $236 \pm 35$ & \& Kerr (1969) \\
\hline 178. & $432 \pm 130^{a}$ & Bennett (1963) & 1414. & $274.7 \pm 0.4$ & Altenhoff et al. (1970) \\
\hline 178. & $400 \pm 80^{a}$ & Bennett (1963) & 1420. & $180 \pm 36^{c}$ & Leslie (1960) \\
\hline 178. & $378 \pm 113^{a}$ & Kellermann et al. (1969) & 442. & $210 \pm 20$ & Giacani et al. (1997) \\
\hline 178. & $378 \pm 113^{a}$ & Holden \& Cas & 1442 & $300 \pm 7$ & This work \\
\hline 195. & $398 \pm 119^{a}$ & Kundu \& Velus & 2650 & $167 \pm 17$ & $\&$ Ker \\
\hline 324 . & $411 \pm 50^{b}$ & This work & 2695. & $170.3 \pm 0.4$ & Altenhoff et al. (1970) \\
\hline $330 .$. & $469 \pm 94^{a}$ & Kassim (1992) & 2700 . & $107 \pm 10$ & Altenhoff et al. (1970) \\
\hline 330. & $400 \pm 40^{a}$ & Giacani et al. (1997) & 2700 . & $169 \pm 18$ & Willis (1973) \\
\hline $400 \ldots$ & $360 \pm 72^{a}$ & Davis et al. (1965) & 2700 . & $179 \pm 10$ & Velusamy \& Kundu (1974) \\
\hline $408 \ldots \ldots$ & $291 \pm 58$ & Large et al. (1961) & $2700 \ldots$ & $124 \pm 19$ & Milne \& Dickel (1974) \\
\hline $408 \ldots \ldots$ & $440 \pm 88$ & Kesteven (1968) & $3000 \ldots$ & $146 \pm 29$ & Scheuer (1963) \\
\hline $408 \ldots \ldots$ & $290 \pm 58$ & Clark et al. (1 & $3125 \ldots \ldots$ & $135 \pm 14$ & Kuz'min et al. ( \\
\hline $430 .$. & $291 \pm 59$ & Kun & $4875 \ldots$ & 123 & et al. (1980) \\
\hline $430 \ldots \ldots$ & $567 \pm 178$ & yer (1975) & $5000 \ldots$ & $127 \pm 15$ & Kundu \& Velusamy (1969) \\
\hline 513. & $297 \pm 30^{c}$ & Kuz'r & 5000. & $155 \pm 23$ & Milne (1969) \\
\hline & $314 \pm 32$ & More & $5000 \ldots$ & $126.7 \pm 0.4$ & Altenhoff et al. (197 \\
\hline & $249 \pm 15^{c}$ & Kuz's & 8350. & $95 \pm 23^{c}$ & Hollinger \& Hobbs (1966) \\
\hline $750 \ldots$ & $242 \pm 24$ & Kellermann et al. (1969) & $10700 \ldots$ & $104 \pm 7$ & Kundu \& Velusamy (1972) \\
\hline
\end{tabular}

a No correction to Baars et al. (1977) scale was applied.

${ }^{b}$ Flux density scale from VLA Calibrator Manual, http:/www. aoc.nrao. edu/ gtaylor/calib.html.

The correction factor was not available. 


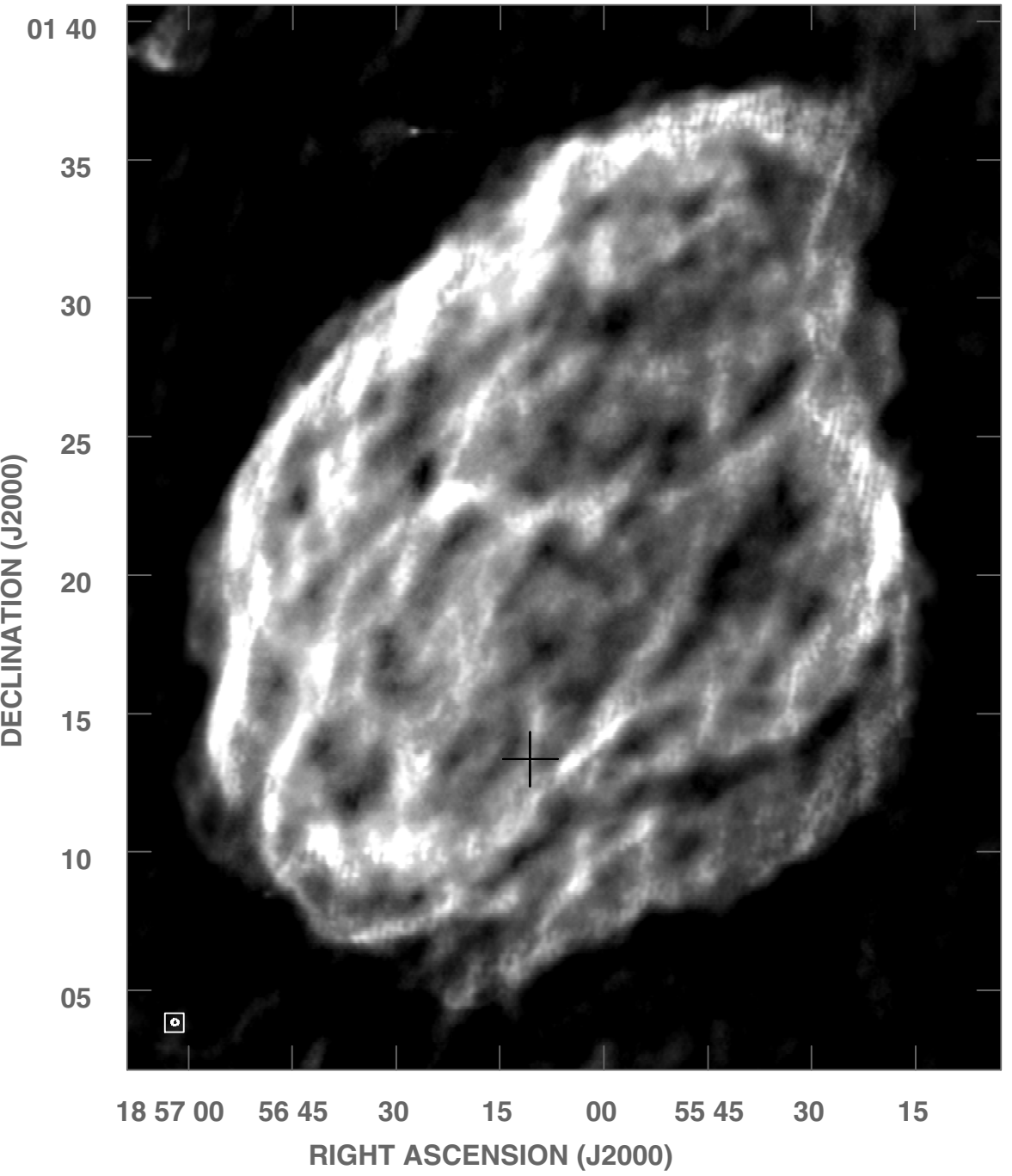

Fig. 4. Image of SNR W44 at $324 \mathrm{MHz}$. The grayscale is linear ranging from 20 to $60 \mathrm{mJy} \mathrm{beam}^{-1}$. The rms noise level is $5.0 \mathrm{mJy}$ beam $^{-1}$. The synthesized beam shown at the bottom left corner is $13^{\prime \prime} \times 13^{\prime \prime}$. The black plus sign indicates the position of the PSR B1853+01.

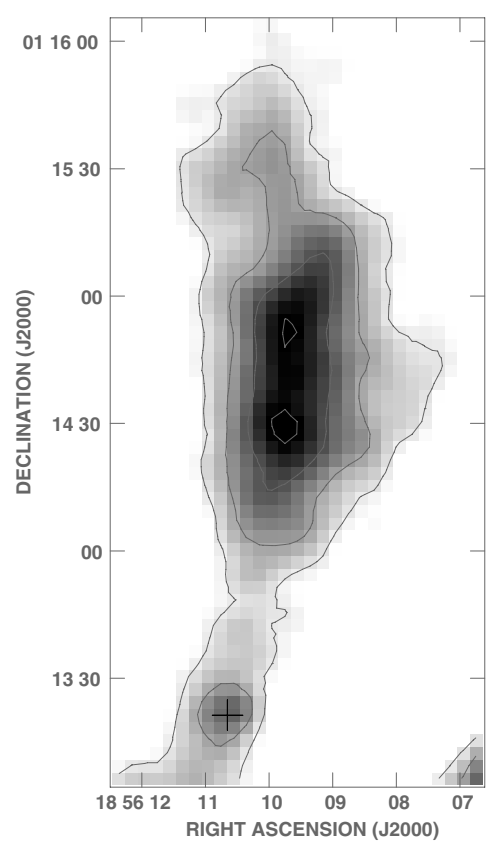

Fig. 5. A close-up image of the pulsar wind nebula around PSR B1853+01 at $324 \mathrm{MHz}$. The position of the pulsar is indicated by the plus sign. The grayscale runs from 34 to $56 \mathrm{mJy} \mathrm{beam}^{-1}$ and the contour levels are $0.036,0.042,0.049,0.056 \mathrm{mJy} \mathrm{beam}^{-1}$.

at 74 and $324 \mathrm{MHz}$ together with the reprocessed VLA data at $1442 \mathrm{MHz}$.

To accurately determine the spectral index distribution based on interferometric radio images, identical beam size and shape at the different pairs of frequencies is required. As mentioned in Sect. 2, we applied appropriate $u v$-tapering, in order to match the range of spatial scales measured at each frequency. In addition, to avoid any positional offsets, the images were aligned and interpolated to identical projections (field center, pixel separation, etc). The final common resolution is $40^{\prime \prime} \times 36^{\prime \prime}, \mathrm{PA}=-62^{\circ}$ for the $74 / 324 \mathrm{MHz}$ comparison and $14^{\prime \prime} \times 14^{\prime \prime}, \mathrm{PA}=-45^{\circ}$ for the $324 / 1442 \mathrm{MHz}$ pair.

The spectral study was carried out by direct comparison of the matched images at the three frequencies and by constructing tomographic images. The different procedures ensure that the observed spectral features are robust, and unaffected by any artifacts or biases in the databases used for the comparison, like zero level differences, smooth background variation, etc.

In spite of the relatively good fidelity attained in the new images, some residual small-scale fluctuations, probably not real, were apparent at the highest spatial resolution traced in the spectral index maps. We therefore preferred to further smooth all three frequency maps to a $50^{\prime \prime}$ resolution, thus avoiding any small scale variation that could mask the main spectral features. We based our analysis on spectral comparisons derived from these smoothed databases. 


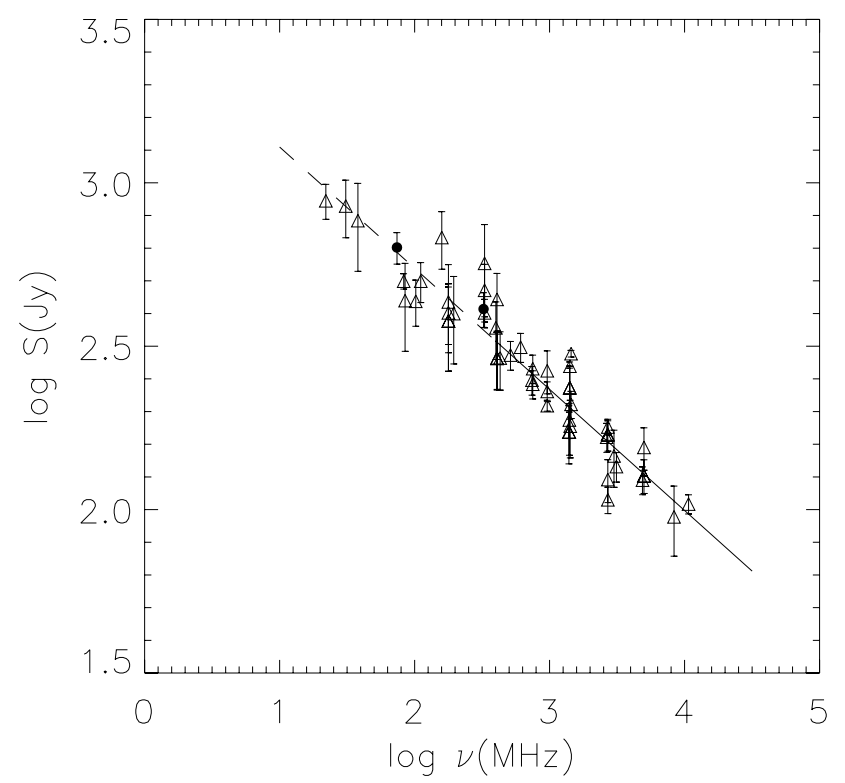

Fig. 6. Integrated radio continuum spectrum of W44 obtained from the flux density values listed in Table 2 . The filled symbols correspond to data from the new VLA measurements at 74 and $324 \mathrm{MHz}$, while the rest of the values were taken from the literature and, where possible, brought onto a single flux density scale. The linear fit to all of the flux density values yields a spectral index $\alpha=-0.37 \pm 0.02\left(S_{v} \propto v^{\alpha}\right)$ and is shown by the line. The dashed part of the line indicates the range of frequencies over which no correction to bring the measured values to the Baars et al. (1977) scale was available.

\subsubsection{Spectral index maps}

Figure 7a shows a $324 \mathrm{MHz}$ continuum image of the SNR W44 smoothed to $25^{\prime \prime}$ resolution for comparison, while Fig. $7 \mathrm{~b}$ and Fig. 7c display the spatial spectral index distribution in the remnant obtained from the direct ratio of the matched $324 / 1442 \mathrm{MHz}$ and $74 / 324 \mathrm{MHz}$ data, at the angular resolution of $50^{\prime \prime}$. To construct these spectral index maps the images were clipped at the $4 \sigma$ level of their respective noise levels. Since, as noted in Sect. 4.1, the global spectrum of W44 remains a power law to well below $100 \mathrm{MHz}$, we use the same scale in Fig. 7 for both frequency pairs in order to easily identify continuum spectral changes that depend on both position and frequency (spectral curvature).

In Fig. $7 b$ it is readily apparent that the brightest radio emission all along the periphery of W44 has a spectral index $\alpha_{324}^{1442} \sim-0.4,-0.5$, compatible with a first-order Fermi mechanism operating at the shock. In addition, the bright filaments observed across the SNR have a similar $\alpha$, in contrast with the slightly flatter diffuse interior.

In the spectral index map traced between 74 and $324 \mathrm{MHz}$ (Fig. 7c) the most striking feature is the short band seen on the southeastern limb (near $18^{\mathrm{h}} 56^{\mathrm{m}} 50^{\mathrm{s}}, 01^{\circ} 17^{\prime}$ ), where the spectral index becomes positive, with $\alpha$ varying between 0 and +0.4 . This spectral inversion occurs at a site where previous studies have demonstrated that the SNR is physically interacting with a dense molecular cloud (Seta et al. 2004; Reach et al. 2005). Also, as shown below (Sect. 5.1) from $24 \mu \mathrm{m}$ and $8 \mu \mathrm{m}$ Spitzer observations, this is the location of an HII region (named G034.700.6 in the HII regions catalog by Paladini et al. 2003, centered at $18^{\mathrm{h}} 56^{\mathrm{m}} 47.9^{\mathrm{s}}, 01^{\circ} 17^{\prime} 54^{\prime \prime} \mathrm{J} 2000$ ) with the IRAS point source $18544+0112$ (identified as a young stellar object) located on its border. Thus, the spectral inversion can be interpreted as originating from low frequency free-free absorption at the
SNR/molecular cloud shock boundary. Absorbing ionized gas could have been created by the passage of a fast, ionizing J-type shock that passed through the molecular cloud and dissociated and ionized the gas. A similar spectral inversion was observed by Brogan et al. (2005) in the SNR 3C 391, where a spectral turnover for frequencies below $100 \mathrm{MHz}$ was found at the positions where the SN shock was running into dense molecular gas. An alternative explanation is that thermal absorption is occuring inside the boundaries of the coincident HII region, along its periphery where the thermal electron density might be highest. We note that this localized absorption has a negligible influence on the total integrated flux and thus has no measurable impact on the integrated continuum spectrum.

From the comparison between Fig. 7b (324/1442 MHz) and Fig. 7c (74/324 MHz) one could have the misleading impression that the spectral behavior is reversed from one frequency range to the other. In fact the spectral index of the limb and bright filaments is the same (near $\sim-0.4$ to -0.5 ) in both frequency pairs, and it is the diffuse interior that changes to a steeper value in the 74/324 MHz comparison. This effect, however, needs to be confirmed on the basis of more sensitive $74 \mathrm{MHz}$ data before advancing an interpretation.

What is readily apparent from the comparison of Figs. 7b and $7 \mathrm{c}$ is that the westernmost arc changes its spectrum from $\alpha_{324}^{1442} \sim-0.4$ to a flatter value $\alpha_{74}^{324} \sim-0.2$. This feature is also located at a site where the $\mathrm{SN}$ shock is probably running into a dense molecular cloud, where the intense near infrared emission (see Sect. 5.1) and the bright optical filaments observed in the [SII] and [NII] lines (Mavromatakis et al. 2003) suggest the presence of radiative shocks. In this case, it is possible that the observed change in the radio continuum spectrum is due to absorption by a layer of thermal electrons associated with the sheets responsible for the filaments.

\subsubsection{Tomographic images}

To investigate the fidelity of our radio continuum spectral index maps, we also produced a tomographic gallery (see for example Katz-Stone \& Rudnick 1997). A tomographic map between two images at different frequencies is made by using a test spectral index $\alpha_{\mathrm{t}}$ to scale the brightness in the higher frequency image $\left(S_{v_{1}}\right)$, and then subtracting this scaled image from the lower frequency image $\left(S_{v_{2}}\right)$. Thus, a tomographic gallery is obtained by calculating $S_{\mathrm{t}}=S_{v_{2}}-\left(v_{2} / v_{1}\right)^{\alpha_{\mathrm{t}}} S_{v_{1}}$, stepping through a range of test spectral indices $\alpha_{\mathrm{t}}$. Features which have a spectral index identical to the test value will disappear in the $S_{t}$ image. Spatial components that have different spectral indices will appear as positive or negative residuals depending upon whether the spectrum is steeper or flatter than the assumed $\alpha_{\mathrm{t}}$ value. Such a procedure allows us to trace fine-scale spectral index changes, and is particularly useful for disentangling the superposition of components with different spectra that can overlap along the line of sight.

In Fig. 8 we present a tomographic gallery of 324 vs. $1442 \mathrm{MHz}$ data, while the comparison between 74 and $324 \mathrm{MHz}$ data is shown in Fig. 9. In both cases the tomographic images were generated for five test spectral index values: $\alpha_{\mathrm{t}}=+0.1$, $\alpha_{\mathrm{t}}=-0.1, \alpha_{\mathrm{t}}=-0.3, \alpha_{\mathrm{t}}=-0.5$, and $\alpha_{\mathrm{t}}=-0.7$. In these maps the positive spatial components (seen as light regions) are steeper in spectral index than the assumed test value, while the negative (dark) residuals correspond to features having a spectrum flatter than the test value. In both figures, the bottom right frame displays the $324 \mathrm{MHz}$ continuum image for comparison. 

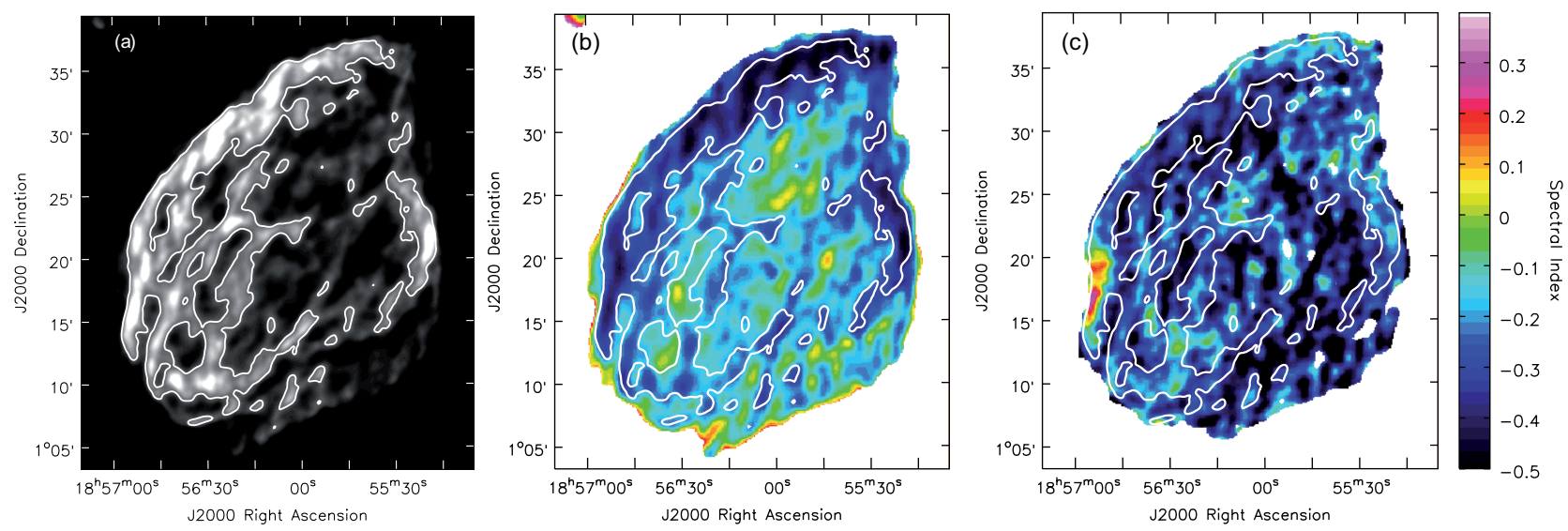

Fig. 7. a) $324 \mathrm{MHz}$ image of W44 with 25" resolution showing the location of the brightest filaments. b) Spectral index map between 324 and $1442 \mathrm{MHz}$ (50" resolution). c) Spectral index map between 74 and $324 \mathrm{MHz}$ (50" resolution). The $0.15 \mathrm{Jy}^{\prime \prime}$ beam ${ }^{-1}$ contour from the $25^{\prime \prime}$ resolution $324 \mathrm{MHz}$ image is included on each panel to facilitate the comparison between spectral continuum and total power features. Only regions with flux densities greater than $4 \sigma$ were used to create the spectral index maps in b) and $\mathbf{c}$ ). Both spectral index maps have the same color scale (displayed to the right).
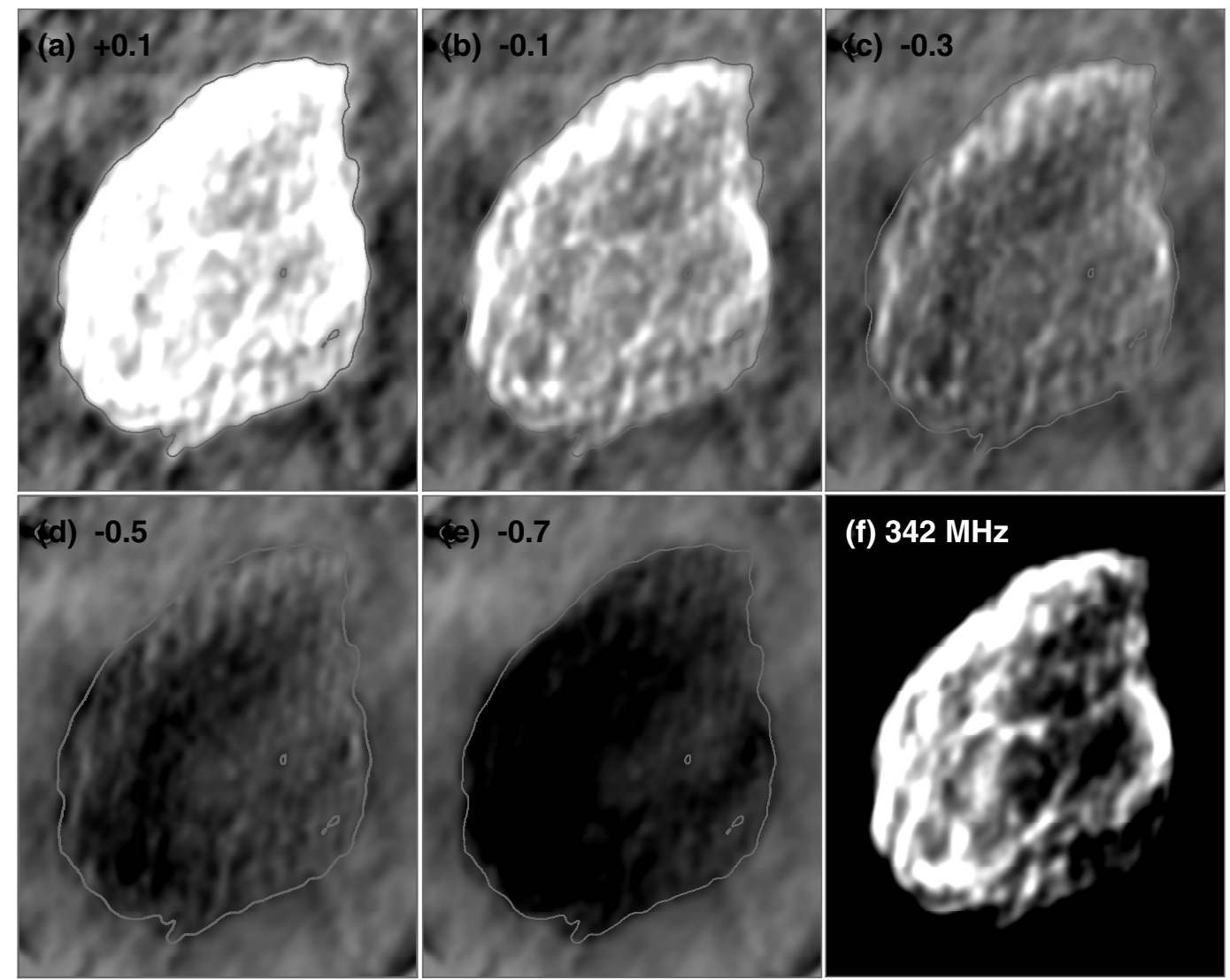

Fig. 8. Gallery of tomographic images for the SNR W44 constructed from data at 324 and $1442 \mathrm{MHz}$, which were matched in uv coverage. The data at both frequencies have a common 50" resolution. The spectral index $\alpha_{\mathrm{t}}$ is indicated at the top left corner of the panels. Bright areas have radio continuum spectral indices steeper than the assumed $\alpha_{\mathrm{t}}$ and dark areas imply flatter spectrum. The grayscale is the same in all panels. A $324 \mathrm{MHz}$ intensity contour at $260 \mathrm{mJy}^{\text {beam }}{ }^{-1}$ is plotted for reference. The image of W44 at $324 \mathrm{MHz}$ plotted for comparison is displayed in the bottom right frame.

In the 74/324 MHz comparison (Fig. 9), the feature with positive spectrum noted earlier near $\sim 18^{\mathrm{h}} 57^{\mathrm{m}}, 01^{\circ} 17^{\prime}$ is strikingly revealed as a short, dark filament in the eastern border of all the tomographic images. It is especially evident in panels from $\alpha_{\mathrm{t}}=-0.1$ to $\alpha_{\mathrm{t}}=-0.3$ as a structure with flatter spectrum than these values. Also, the tomographies traced between
74 and $324 \mathrm{MHz}$ emphasize the westernmost arc, that stands out as a flatter spectral index feature in the images traced at $\alpha_{\mathrm{t}}=-0.5$ and $\alpha_{\mathrm{t}}=-0.7$ and disappears against the background at $\alpha_{\mathrm{t}}=-0.3$, while for the tomographies traced between 324 and $1442 \mathrm{MHz}$ (Fig. 8) the best fit is attained for $\alpha_{\mathrm{t}}$ between -0.5 and 

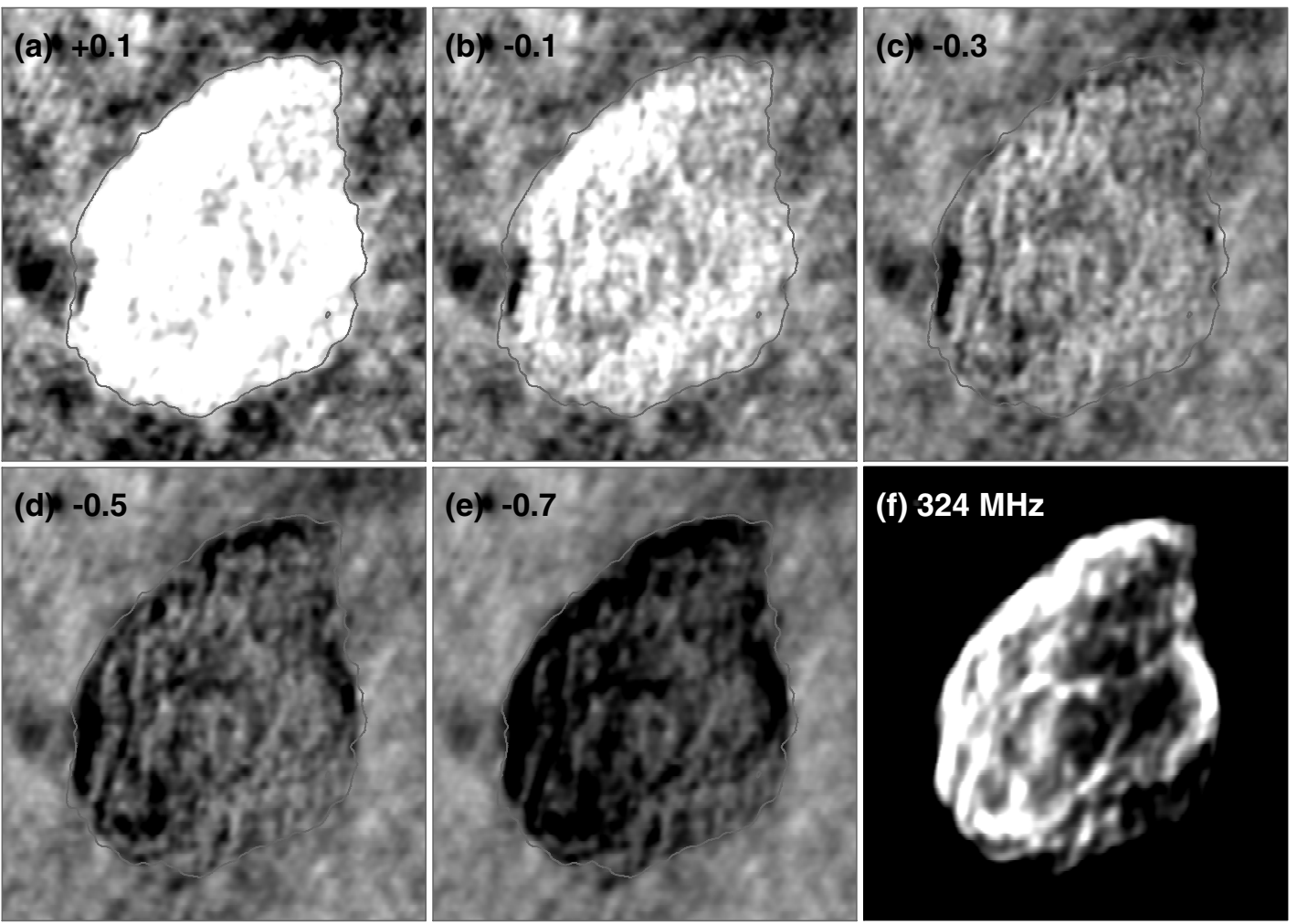

Fig. 9. The same as Fig. 8 but between 74 and $324 \mathrm{MHz}$. Bright areas have radio spectral indices steeper than the assumed $\alpha_{\mathrm{t}}$ and dark areas imply flatter spectral indices. The greyscale is the same for each spectral index panel. The $324 \mathrm{MHz}$ contour at $140 \mathrm{mJy} \mathrm{beam}^{-1}$ is also plotted in each panel for reference. A greyscale $324 \mathrm{MHz}$ image with a range of 26 to $70 \mathrm{mJy} \mathrm{beam}^{-1}$ is shown in the lower right panel for comparison.

-0.7 , thus confirming the negative (concave down) curvature of the spectrum recognized earlier.

In summary, all of the important radio continuum spectral index characteristics discussed in the previous section are confirmed through this alternative analysis.

An important conclusion from this new radio continuum spectral study is that neither spectral index maps nor tomographies reveal any trace of coupling between the pulsar and the surrounding plasma beyond the PWN; that is, there is no spectral signature, such as a smooth radial steepening, that would suggest a progressive aging of particles from their injection near the pulsar to their transport further out into the SNR shell. Moreover, the spectral index obtained for the shell $(\alpha \sim-0.5)$, compatible with the diffusive shock acceleration mechanism in the test particle limit, confirms that the relativistic electrons responsible for the shell radio emission are accelerated right at the shock front, with no influence from the central neutron star. Also, no spectral signature is observed that can be related to the presence of the $95 \%$ confidence ellipse corresponding to the EGRET $\gamma$-ray source that covers most of the southeast corner of W44.

\section{Multi-wavelength comparisons}

\subsection{Radio, infrared and molecular emission}

Recently, Reach et al. (2006) used Spitzer Space Telescope GLIMPSE data to resolve the mid-infrared structure of W44 between 3.6 and $8 \mu \mathrm{m}$ (Benjamin et al. 2003). To study the relationship between the infrared and radio features in the SNR $\mathrm{W} 44$ we have combined the data at $4.5 \mu \mathrm{m}$ with the new radio image at $324 \mathrm{MHz}$ in a false color image (Fig. 10). In this figure, the mid-infrared emission is shown in orange and the radio emission in blue; magenta thus corresponds to regions where both spectral bands overlap. The distribution of compact $\mathrm{OH}$ (1720 MHz) maser spots (Claussen et al. 1997; Hoffman et al. 2005) are shown as plus signs.

The mid-IR (MIR) image reveals extensive filamentary emission and some isolated bright clumps. This emission is particularly bright in the north and southwestern parts of W44, where bright near infrared $\mathrm{H}_{2}$ line $(2.12 \mu \mathrm{m})$ emission is also present (Reach et al. 2005). Figure 10 reveals the impressive agreement between the low frequency radio emission and the infrared $4.5 \mu \mathrm{m}$ emission to the north and southwest, where the brightest radio filaments are almost exactly coincident with the infrared radiation. In contrast, the central and eastern regions do not show much obvious correspondence with the exception of a few bright knots. The westernmost bright radio arc near $18^{\mathrm{h}} 55^{\mathrm{m}} 20^{\mathrm{s}} 01^{\circ} 22^{\prime}$, which is also bright in $\mathrm{H}_{\alpha}$, [SII] and [NII] lines (Giacani et al. 1997; Mavromatakis et al. 2003), has an infrared counterpart that mimics the appearance of the radio arc.

The mid-IR study is also useful for understanding W44 in the context of the surrounding interstellar medium. Figure 11 displays a three color image of W44 comparing the radio morphology at $324 \mathrm{MHz}$ (blue) with Spitzer GLIMPSE and MIPSGAL images at $8 \mu \mathrm{m}$ (green) and $24 \mu \mathrm{m}$ (red), respectively (Carey et al., in preparation). These infrared bands are excellent tracers of the warm dust associated with star formation. The most conspicuous feature in Fig. 11 is the $\sim 10$ arcmin region just outside the southeast limb of W44 (red nebula), identified by Paladini et al. (2003) as the HII region G034.7-00.6. In this case the combination of the 8 and $24 \mu \mathrm{m}$ images reveals with impressive detail the hot dust grains (in red) in the Strömgren sphere, limited to the east by an annular photo dissociation region (PDR) dominated by polycyclic aromatic hydrocarbon (PAH) emitting near $8 \mu \mathrm{m}$ (green). The bright yellow spot abuts the only portion 


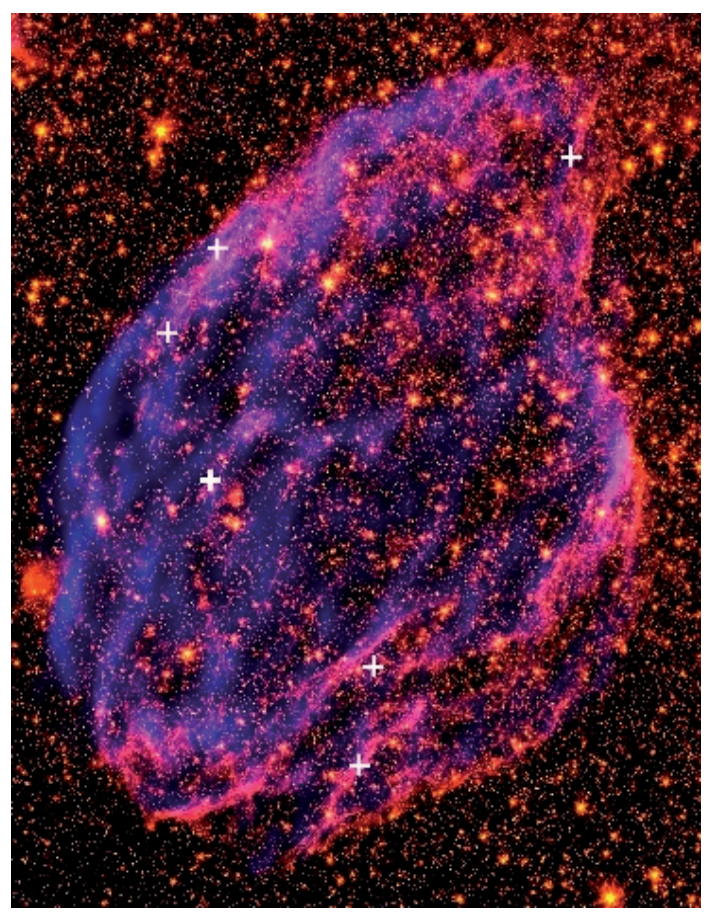

Fig. 10. A color composite image showing the spatial correlation between the mid-infrared emission as observed by the Spitzer Space Telescope at $4.5 \mu \mathrm{m}$ (in orange) from Reach et al. (2006), and the new low frequency radio image at $324 \mathrm{MHz}$ (in blue). Features where both spectral bands overlap are magenta in color. The positions where $\mathrm{OH}$ $(1720 \mathrm{MHz})$ maser emission was detected are indicated with white + symbols (Hoffman et al. 2005).

of the eastern limb of W44 with a concave shape (near $\sim 18^{\mathrm{h}} 57^{\mathrm{m}}$, $01^{\circ} 17^{\prime}$ ) coincident with the brightest radio filament and the region determined to have a positive (inverted) radio spectrum (as shown in Sect. 4.2). The IR point source is the IRAS source $18544+0112$, proposed by Molinari et al. (1996) to be a young stellar object (YSO) in a primitive evolutionary stage.

As summarized in the introduction (Sect. 1), W44 is one of the few SNRs known to be physically interacting with its parent molecular cloud complex, with high-angular resolution molecular studies in different near-IR (NIR) and millimetric lines (see for example Seta et al. 2004; Reach et al. 2005) clearly indicating its evolution within a giant molecular cloud. In particular, one molecular cloud component has been detected adjacent and closely parallel to its bright eastern radio boundary (Reach et al. 2005, see for instance Fig. 3e in their paper), while another one is located near the very bright western arc. Seta et al. (2004) provide a useful schematic illustration of the various $\mathrm{CO}$ features discovered towards W44 (Fig. 3 in their paper). In particular, and near the radial velocity of $\sim 47 \mathrm{~km} \mathrm{~s}^{-1}$ approximately corresponding to the $2.9 \mathrm{kpc}$ distance attributed to W44, they identify a CO "edge" feature exactly at the position of the YSO and the indentation of the eastern rim (E2 in their nomenclature). Based on the abrupt changes observed in the physical parameters, Seta et al. (2004) interpret the SNR as interacting with this particular molecular cloud component.

In this scenario involving molecular gas, an SNR, an HII region, and a YSO, it is worthwile to ask whether this may be a case of star formation triggered by the action of a $\mathrm{SN}$ shock on a coincident molecular cloud or, alternatively, whether it might illustrate a case of sequential star formation at the periphery of the HII region. This last case has been, for example, observed in the Sh 217 and Sh 219 HII regions (Deharveng et al. 2003).
Although Molinari et al. (1996) proposed a kinematic distance of $3.7 \mathrm{kpc}$ for the YSO, this distance might be an overestimate and the YSO is instead co-distant with W44, at about $2.9 \mathrm{kpc}$. The question then is whether the age of W44, of a few $10^{4} \mathrm{yrs}$, is sufficient to have been responsible for the formation of new, massive stars. The timescale of star formation is currently under ample debate, but it is generally agreed that a few $10^{6}$ yrs are necessary for the formation of protostars from condensed molecular matter (e.g. Tassis \& Mouschovias 2004, and references therein). We are therefore led to the conclusion that if the YSO is related to W44, then the cloud collapse must have been initiated by the action of the precursor star's wind and not directly by the SNR. Therefore we feel it is more plausible that the YSO is associated with the PDR around the HII region G034.7-00.6. Detailed NIR, MIR and millimeter studies are planned to further investigate the star formation in this complex region, with the goal of uncovering the underlying genetic links between the SN shock, the HII region, and the protostar.

From Fig. 11 regions of more nebulous infrared emission toward the north-northwestern sides of W44 are also apparent, and the yellow spot about $\sim 1$ arcmin in size seen to the east of its geometrical center corresponds to the HII region G034.7-00.5 (centered at $18^{\mathrm{h}} 56^{\mathrm{m}} 26.5^{\mathrm{s}}, 01^{\circ} 20^{\prime} 38^{\prime \prime}$, Paladini et al. 2003). Observations of radio recombination lines (RRL) would clearly be helpful to further elucidate the presence of thermally emitting gas in this area.

\subsection{Radio and $X$-ray emission}

The X-ray emission from W44 has been investigated using the Einstein, EXOSAT, ROSAT, ASCA, and Chandra telescopes (Smith et al. 1985; Jones et al. 1993; Rho et al. 1994; Harrus et al. 1996; Shelton et al. 2004). In Fig. 12a we present a comparison between the $324 \mathrm{MHz}$ radio image and the X-ray emission as observed with ROSAT in the energy range 0.2 to $2.4 \mathrm{keV}$ (taken from ROSAT archives). As reported by Rho \& Petre (1998), the brightest X-ray features are preferentially located in the center of the remnant, in a region of relatively low radio surface brightness. As expected, no correspondence is observed between the mostly thermal X-ray emission (Shelton et al. 2004) and the filamentary synchrotron radio emission. A number of physical processes such as cloud evaporation, electron thermal conduction, and entropy mixing have been proposed to explain the observed X-ray properties (see Shelton et al. 2004, for a review of the models). Reach et al. (2005) conclude that probably both, evaporating clumps and thermal conduction behind radiative shocks, are operating to produce the observed X-ray radiation.

Figure 12b shows the Chandra image of W44 produced by Shelton et al. (2004) from the combination of 6 different pointings with the outer contour (traced at $4 \sigma$ level) of the $324 \mathrm{MHz}$ radio emission plotted for comparison. Shelton et al. (2004) suggest that diffuse X-ray emission is observed extending slightly beyond the northwestern border of the radio synchrotron emission. The new high-sensitivity radio images presented here, however, do not support this conclusion.

\section{Energetics of SNR W44}

As W44 harbours a pulsar with a PWN and is spatially coincident with the GeV gamma-ray source 3EG 1856+0114, it is useful to calculate its total energy content and compare it with other Galactic SNRs with central compact objects. The total synchrotron energy is stored in the magnetic field as well as in the 

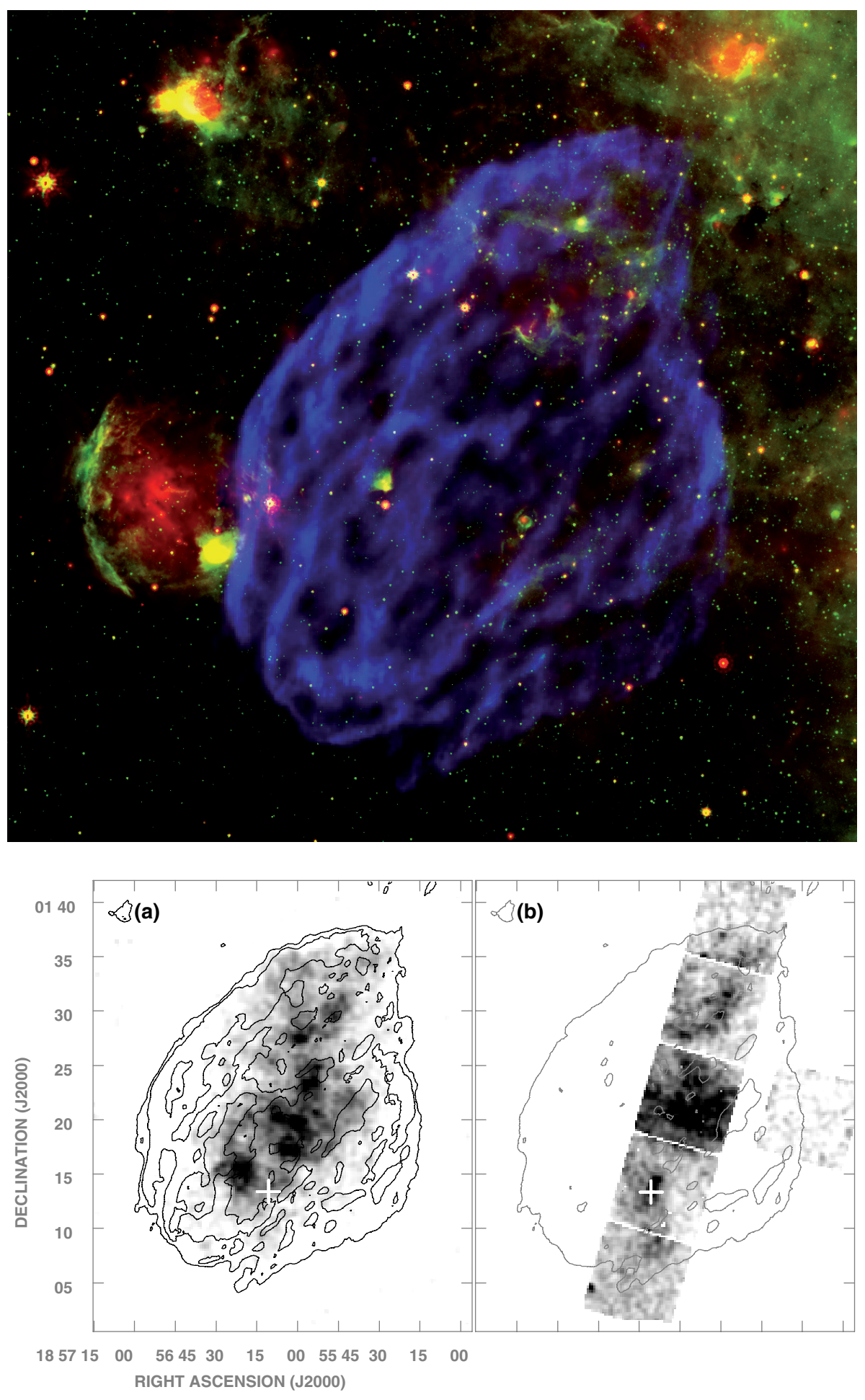

Fig. 11. A high resolution comparison of the radio continuum emission at $324 \mathrm{MHz}$ of the W44 and the infrared emission in the SNR region. The green and red images corresponds to the Spitzer 8 and $24 \mu \mathrm{m}$ data, while in blue the $324 \mathrm{MHz}$ image of the remnant with $13^{\prime \prime}$ resolution is shown.

Fig. 12. An X-ray/radio comparison of the SNR W44. a) The grayscale corresponds to the thermal X-ray emission observed with ROSAT in the $0.2-2.4 \mathrm{KeV}$ range, while the overlaid contours trace $324 \mathrm{MHz}$ radio emission at 20 and $50 \mathrm{mJy} \mathrm{beam}^{-1}$. b) Chandra $0.7-2.6 \mathrm{keV}$ image of W44 (as taken from Shelton et al. 2004) with the $20 \mathrm{mJy}^{\text {beam }}{ }^{-1}(4 \sigma)$ radio contour at $324 \mathrm{MHz}$ superimposed for comparison. The white plus sign shows the position of the pulsar PSR B1853+01.

kinetic energy of the relativistic particles. Under the assumption of equipartition between particles and magnetic energy, the minimum energy content and the corresponding magnetic field can be obtained from the following relations (Moffet 1975)

$U_{\min }=0.50(a A L)^{4 / 7} V^{-3 / 7}$

$B_{\text {min }}=2.3(a A L / V)^{2 / 7}$

where $V$ is the volume of the source, $A$ is a factor which depends on the assumed lower and upper cutoff frequencies and the spectral index, and $L$ is the total luminosity of the source. We note that electrons may not be the only energetic particles within the source; an appreciable amount of energy may also be stored in energetic baryons. The total particle energy can be described as $U_{\mathrm{p}}=a U_{\mathrm{e}}$, where $a$, the energy ratio between electrons and baryons is usually assumed to be $\sim 50$ (Moffet 1975).

Using the integrated spectral index of -0.37 and assuming isotropic radiation, we derive a total luminosity for W44 of $7.8 \times 10^{34} \mathrm{erg} \mathrm{s}^{-1}$. The volume of the source is estimated to be about $2 \times 10^{59} \mathrm{~cm}^{3}$ by approximating W44 as a sphere of radius $11.6 \mathrm{pc}$. For typical lower and upper cutoff frequencies of $10^{7}$ and $10^{11} \mathrm{MHz}$, the total minimum energy content is 
$5.8 \times 10^{49} \mathrm{erg}$. Following the same procedure and with similar assumptions we also re-estimated the total energy content of the PWN based on the $324 \mathrm{MHz}$ data. We derive its minimum energy as $U_{\min }(P W N)=1.2 \times 10^{47} \mathrm{erg}$, in good agreement with Frail et al. (1996)'s results $\left(U_{\min } \simeq 1 \times 10^{47} \mathrm{erg}\right)$ estimated from higher radio frequencies observations.

The minimum energy derived for W44 is comparable to the value obtained for SNR CTB80 $\left(U_{\min }=5 \times 10^{49} \mathrm{erg}\right.$, Castelletti et al. 2003), another SNR containing a pulsar, and two orders of magnitude higher than the energy content of $\mathrm{W} 28\left(U_{\min }=\right.$ $2 \times 10^{47}$ erg, Dubner et al. 2000).

The corresponding minimum magnetic field in the SNR is $B_{\text {min }}=18 \mu \mathrm{G}$, consistent with the compression of a typical ISM magnetic field (about $5 \mu \mathrm{G}$, Wielebinski \& Beck 2005) by a factor of three to four.

\section{Conclusions}

We have presented new high-sensitivity and high-resolution low frequency VLA radio images of the SNR W44. These images provide an unprecedented view of W44 with an angular resolution of $\sim 37^{\prime \prime}$ and $\sim 13^{\prime \prime}$ at 74 and $324 \mathrm{MHz}$, respectively, an improvement of over two orders of magnitude over the best, previous low-frequency image of this SNR.

Based on our $74 \mathrm{MHz}$ image sensitive to structures with angular scales from a few arcseconds to $\sim$ half a degree, we measure an integrated flux density for W44 of $S_{74 \mathrm{MHz}} \simeq 634 \mathrm{Jy}$. Similarly, from our $324 \mathrm{MHz}$ image sensitive to spatial structures between $\sim 13$ arcsec and $\sim 1$ degree, we derive an integrated flux density $S_{324 \mathrm{MHz}} \simeq 411 \mathrm{Jy}$. We have used these measurements, together with carefully considered flux densities present in the literature, to calculate an accurate global radio spectrum of the SNR. The best fit to the data yields an integrated spectral index $\alpha=-0.37 \pm 0.02$, constant over four decades in frequency with no significant turnover down to $74 \mathrm{MHz}$. The straight power law spectrum is in contrast to a number of other SNRs that exhibit spectral turnovers at low frequencies, and is consistent with previous low frequency measurements of this object.

The new 74 and $324 \mathrm{MHz}$ data, together with existing $1442 \mathrm{MHz}$ data, were combined to perform the first careful, spatially resolved study of the synchrotron spectrum in W44. Highdynamic range observations at three well spaced frequencies are ideal for accurately constraining the variation of the spectrum within the source. Our results were obtained first from standard analysis of spectral index maps, and thereafter confirmed using spectral tomography techniques. The main findings of our radio continuum spectral index study are:

(i) The eastern limb, which is the brightest portion of W44 SNR, as well as the narrow filaments observed in total power across most of the surface of the source have a straight power law spectrum between 74 and $1442 \mathrm{MHz}$ with $\alpha \sim-0.4,-0.5$. This result is consistent with the predictions of a simple, diffusive shock acceleration model applied to the filamentary shell of W44.

(ii) From the $74 / 324 \mathrm{MHz}$ comparison we have discovered a narrow feature with a positive spectrum $(0.0<\alpha<+0.4)$ located towards the southeast border of W44, coincident with an indentation in the limb of the SNR. At this location existing studies have shown that the SNR shock is interacting with an external molecular cloud. Also, flanking this portion of W44, mid-IR observations have revealed the presence of an almost circular HII region surrounded by a PDR region. Exactly at the interface between this HII region and W44, as seen in the plane of the sky, a young stellar object is present. A likely explanation of the inversion in the low frequency radio continuum spectrum in this region is free-free absorption from ionized gas in the post shock region at the $\mathrm{SNR} /$ molecular cloud interface. If this is the case, it marks the second case of spatially resolved low frequency radio observations tracing an $\mathrm{SNR} /$ molecular cloud interaction, following Brogan et al. (2005)'s detection of thermal absorption at the periphery of 3C 391. An alternative explanation is that the thermal absorption is occuring at the borders of the cavity carved by the collindant HII region, where the column density of thermal electrons might be highest. The spectral inversion is probably produced by a combination of both these effects. However this localized absorption is negligible compared to the total integrated flux and thus has no measurable influence on the integrated continuum spectrum. Further IR and millimeter observations are planned to investigate the $3 \mathrm{D}$ distribution of the gas emitting in the different spectral regimes, and to elucidate the precise physical processes acting at this site. This should shed light on the complex chain of interconnected events that can tie together the evolution of W44, its parent molecular cloud, precursor star, and neighbouring HII region in a scenario consistent with the formation of a new generation of stars exactly at the interface $\mathrm{SNR} /$ molecular cloud.

(iii) The comparison of the spectrum between 74 and $324 \mathrm{MHz}$ and between 324 and $1442 \mathrm{MHz}$ revealed a spectral flattening ocurring on the westernmost arc of W44 at the lowest frequency pair (concave down spectrum). Our hypothesis is that this spectral flattening is related to the SNR shock colliding with a molecular cloud in this region, consistent with the bright optical filaments and IR emission observed there. In this scenario the spectral flattening could be a consequence of strong postshock densities and enhanced magnetic fields. If the electron energy spectrum hardens at lower frequencies, regions in which the magnetic field is higher than the surroundings will appear both brighter and with a flatter spectrum. A firm estimate of the distribution of magnetic field strength throughout the SNR would be important to test this hypothesis.

(iv) The present study revealed that there is no evidence in the radio continuum spectrum of any coupling between the associated pulsar PSR B1853+01 and the surrounding SNR shell (that could, for example, have been observable as a gradual steepening from the pulsar to the shell). Furthermore, there is no spectral signature indicating any link to the EGRET $\gamma$-ray source whose $95 \%$ confidence ellipse covers most of the southeast corner of W44.

We have also used the new $324 \mathrm{MHz}$ image to perform the first detailed low frequency radio/IR comparison for W44. Bright filamentary IR emission detected by Spitzer at $4.5 \mu \mathrm{m}$ shows an impressive correspondence with the radio emission towards the north and western portions of the remnant. To the central and eastern regions of W44, however, almost no IR counterpart is observed for the radio emission.

From the comparison of the new radio image at $324 \mathrm{MHz}$ with the X-ray emission as seen by ROSAT between 0.2 and $2.4 \mathrm{keV}$ and with a high-resolution Chandra image, we can confirm that the synchrotron radio emission appears to surround the thermal X-ray plasma. As determined from previous studies, the $\mathrm{X}$-ray emission is probably related to a combination of the evaporation of clouds and thermal conduction occuring behind radiative shocks. In addition heavy absorption likely produced by foreground molecular clouds may explain the absence of X-ray radiation towards the southeast.

We have also investigated the energetics in W44 based on the new observations and accurate, re-derived integrated spectrum. 
We find that the total minimum energy content is $5.8 \times 10^{49} \mathrm{erg}$, a value in agreement with the estimates for other SNRs containing central pulsars. The minimum, equipartition magnetic field estimate is $18 \mu \mathrm{G}$.

Finally, we note that this study adds to a growing list of examples confirming the importance of high resolution, low frequency radio observations in providing an important new tool to demonstrate and understand the physical interaction between SNR shocks and the surrounding dense material. This also points to the great potential of an emerging generation of much more sensitive low frequency instruments.

Future work will involve a detailed comparison of the radio spectral index distribution and the molecular emission in order to refine the analysis of the interaction between W44 and its parent molecular cloud. New IR and optical studies are also planned in order to improve our understanding of the underlying genetic links and causal relationships between the evolution of the SNR and the adjacent YSO in this inner Galactic complex.

Acknowledgements. We thank W. Reach for supplying us with the IR data in electronic form. We also acknowledge the referee for his constructive comments. This research has been funded by Argentina grants ANPCYT-PICT 04-14018, UBACYT A055/04, ANPCYT-PICT 03-11235 and PIP-CONICET 6433. Basic research in Radio Astronomy at the Naval Research Laboratory is supported by 6.1 base funding. Data processing was carried out using the HOPE PC cluster at IAFE.

\section{References}

Altenhoff, W. J., Downes, D., Goad, L., Maxwell, A., \& Rinehart, R. 1970, A\&AS, 1, 319

Anderson, M. C., \& Rudnick, L. 1993, ApJ, 408, 514

Baars, J. W. M., Genzel, R., Pauliny-Toth, I. I. K., \& Witzel, A. 1977, A\&A, 61, 99

Beard, M., \& Kerr, F. J. 1969, Australian Journal of Physics, 22, 121

Benjamin, R. A., Churchwell, E., Babler, B. L., et al. 2003, PASP, 115, 953

Bennett, A. S. 1963, MNRAS, 127, 3

Brogan, C. L., Lazio, T. J., Kassim, N. E., \& Dyer, K. K. 2005, AJ, 130, 148

Buckley, J. H., Akerlof, C. W., Carter-Lewis, D. A., et al. 1998, A\&A, 329, 639

Castelletti, G., Dubner, G., Golap, K., et al. 2003, AJ, 126, 2114

Castelletti, G., Dubner, G., Golap, K., \& Goss, W. M. 2006, A\&A, 459, 535

Caswell, J. L., Murray, J. D., Roger, R. S., Cole, D. J., \& Cooke, D. J. 1975, A\&A, 45, 239

Clark, D. H., Green, A. J., \& Caswell, J. L. 1975, Austr. J. Phys. Astrophys. Suppl., 75

Claussen, M. J., Frail, D. A., Goss, W. M., \& Gaume, R. A. 1997, ApJ, 489, 143

Condon, J. J., Cotton, W. D., Greisen, E. W., et al. 1998, AJ, 115, 1693

Cornwell, T., \& Fomalont, E. B. 1999, in ASP Conf. Ser. 180, Synthesis Imaging in Radio Astronomy II, ed. G. B. Taylor, C. L. Carilli, \& R. A. Perley, 187

Cornwell, T. J., \& Perley, R. A. 1992, A\&A, 261, 353

Cotton, W. D., Condon, J. J., Perley, R. A., et al. 2004, in Ground-based Telescopes, ed. J. M., Jr. Oschmann, Proc. SPIE, 5489, 180

Davis, M. M., Gelato-Volders, L., \& Westerhout, G. 1965, Bull. Astron. Inst. Netherlands, 18, 42

de Jager, O. C., \& Mastichiadis, A. 1997, ApJ, 482, 874

Deharveng, L., Zavagno, A., Salas, L., et al. 2003, A\&A, 399, 1135

Dickel, J. R., \& Denoyer, L. K. 1975, AJ, 80, 437

Downes, D., Wilson, T. L., Bieging, J., \& Wink, J. 1980, A\&AS, 40, 379

Dubner, G. M., Velázquez, P. F., Goss, W. M., \& Holdaway, M. A. 2000, AJ, 120,1933

Edge, D. O., Shakeshaft, J. R., McAdam, W. B., Baldwin, J. E., \& Archer, S. 1958, Mem. R. Astr. Soc., 68, 37

Eisenhauer, F., Genzel, R., Alexander, T., et al. 2005, ApJ, 628, 246
Esposito, J. A., Hunter, S. D., Kanbach, G., \& Sreekumar, P. 1996, ApJ, 461, 820

Fatuzzo, M., \& Melia, F. 2005, ApJ, 630, 321

Feast, M., \& Whitelock, P. 1997, MNRAS, 291, 683

Frail, D. A., Giacani, E. B., Goss, W. M., \& Dubner, G. 1996, ApJ, 464, L165

Gaensler, B. M. 1998, ApJ, 493, 781

Giacani, E. B., Dubner, G. M., Kassim, N. E., et al. 1997, AJ, 113, 1379

Harris, D. E. 1962, ApJ, 135, 661

Harrus, I. M., Hughes, J. P., \& Helfand, D. J. 1996, ApJ, 464, L161

Hoffman, I. M., Goss, W. M., Brogan, C. L., \& Claussen, M. J. 2005, ApJ, 627, 803

Holden, D. J., \& Caswell, J. L. 1969, MNRAS, 143, 407

Hollinger, J. P., \& Hobbs, R. W. 1966, Science, 153, 1633

Jones, L. R., Smith, A., \& Angellini, L. 1993, MNRAS, 265, 631

Kassim, N. E. 1989a, ApJ, 347, 915

Kassim, N. E. 1989b, ApJS, 71, 799

Kassim, N. E. 1992, AJ, 103, 943

Kassim, N. E., Perley, R. A., Erickson, W. C., \& Dwarakanath, K. S. 1993, AJ, 106,2218

Katz-Stone, D. M., \& Rudnick, L. 1997, ApJ, 479, 258

Kellermann, K. I., Pauliny-Toth, I. I. K., \& Williams, P. J. S. 1969, ApJ, 157, 1

Kesteven, M. J. L. 1968, Australian Journal of Physics, 21, 369

Kovalenko, A. V., Pynzar', A. V., \& Udal'Tsov, V. A. 1994, Astron. Rep., 38, 784

Kundu, M. R., \& Velusamy, T. 1967, Ann. Astrophys., 30, 723

Kundu, M. R., \& Velusamy, T. 1969, ApJ, 155, 807

Kundu, M. R., \& Velusamy, T. 1972, A\&A, 20, 237

Kuz'min, A. D. 1962, Soviet Astron., 5, 692

Kuz'min, A. D., Levchenko, M. T., Noskova, R. I., \& Salomonovich, A. E. 1960, AZh, 37, 975

Lacey, C. K., Lazio, T. J. W., Kassim, N. E., et al. 2001, ApJ, 559, 954

Large, M. I., Mathewson, D. S., \& Haslam, C. G. T. 1961, MNRAS, 123, 113

Leslard, R. W. 1995, in Proc. Int. Cosmic Ray Conf., Rome, 2, 475

Leslie, P. R. R. 1960, The Observatory, 80, 23

Mavromatakis, F., Boumis, P., \& Goudis, C. D. 2003, A\&A, 405, 591

Mills, B. Y., Slee, O. B., \& Hill, E. R. 1958, Austr. J. Phys., 11, 360

Milne, D. K. 1969, Austr. J. Phys., 22, 613

Milne, D. K., \& Dickel, J. R. 1974, Austr. J. Phys., 27, 549

Moffet, A. T. 1975, Strong Nonthermal Radio Emission from Galaxies: Galaxies and the Universe, ed. A. Sandage, M. Sandage, \& J. Kristian (Chicago: Univ. Chicago Press), 211

Molinari, S., Brand, J., Cesaroni, R., \& Palla, F. 1996, A\&A, 308, 573

Moran, M. 1965, MNRAS, 129, 447

Paladini, R., Burigana, C., Davies, R. D., et al. 2003, A\&A, 397, 213

Pauliny-Toth, I. I. K., Wade, C. M., \& Heeschen, D. S. 1966, ApJS, 13, 65

Petre, R., Kuntz, K. D., \& Shelton, R. L. 2002, ApJ, 579, 404

Radhakrishnan, V., Goss, W. M., Murray, J. D., \& Brooks, J. W. 1972, ApJS, 24, 49

Reach, W. T., Rho, J., \& Jarrett, T. H. 2005, ApJ, 618, 297

Reach, W. T., Rho, J., Tappe, A., et al. 2006, AJ, 131, 1479

Reich, W., Reich, P., \& Fuerst, E. 1990, A\&AS, 83, 539

Reynolds, S. P., \& Ellison, D. C. 1992, ApJ, 399, L75

Rho, J., \& Petre, R. 1998, ApJ, 503, L167

Rho, J., Petre, R., Schlegel, E. M., \& Hester, J. J. 1994, ApJ, 430, 757

Roger, R. S., Costain, C. H., \& Stewart, D. I. 1986, A\&AS, 65, 485

Rowell, G. P., Naito, T., Dazeley, S. A., et al. 2000, A\&A, 359, 337

Scheuer, P. A. G. 1963, The Observatory, 83, 56

Seta, M., Hasegawa, T., Dame, T. M., et al. 1998, ApJ, 505, 286

Seta, M., Hasegawa, T., Sakamoto, S., et al. 2004, AJ, 127, 1098

Shelton, R. L., Kuntz, K. D., \& Petre, R. 2004, ApJ, 611, 906

Smith, A., Jones, L. R., Watson, M. G., et al. 1985, MNRAS, 217, 99

Tassis, K., \& Mouschovias, T. C. 2004, ApJ, 616, 283

Thompson, D. J., Bertsch, D. L., Dingus, B. L., et al. 1996, ApJS, 107, 227

Velusamy, T., \& Kundu, M. R. 1974, A\&A, 32, 375

Westerhout, G. 1958, Bull. Astron. Inst. Netherlands, 14, 215

Wielebinski, R., \& Beck, R. 2005, in LNP Vol. 664: Cosmic Magnetic Fields, ed. R. Wielebinski, \& R. Beck

Willis, A. G. 1973, A\&A, 26, 237

Wilson, R. W. 1963, AJ, 68, 181

Wolszczan, A., Cordes, J. M., \& Dewey, R. J. 1991, ApJ, 372, L99 